\title{
DEFINING A COMPLEX WORKFLOW IN A RESOURCE Allocation Simulation for Mass Data Processing
}

\author{
Zeljko Seremet \& Eugen Mudnic
}
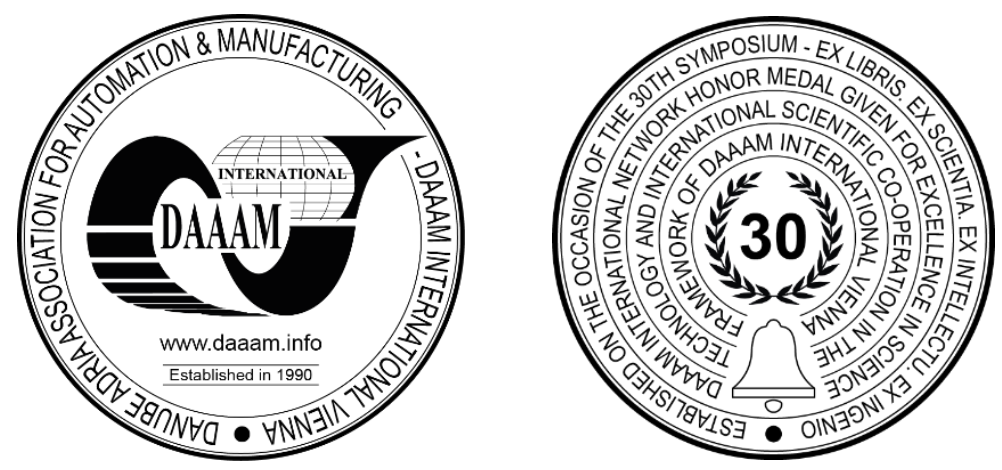

This Publication has to be referred as: Seremet, Z[eljko] \& Mudnic, E[ugen] (2020). Defining a Complex Workflow in a Resource Allocation Simulation for Mass Data Processing, Proceedings of the 31st DAAAM International Symposium, pp.0151-0158, B. Katalinic (Ed.), Published by DAAAM International, ISBN 978-3-902734-29-7, ISSN 1726-9679, Vienna, Austria

DOI: $10.2507 / 31$ st.daaam.proceedings.020

\begin{abstract}
In recent years, the use of mass data processing systems (MDPS) has increased rapidly, both for the processing of research and business data. On the one hand, there is the possibility of buying, pooling, or renting MDPS resources in a scalable and flexible way. On the other hand, there is mass data processing with a certain processing time frame and limited resources to use MDPS resources. As data processing can be mapped to a number of different models of using MDPS resources, it is necessary to be able to assess their adequacy for a given processing, in order to minimize the engagement, i.e. the cost of resources. Such a possibility is provided through processing simulation, which is most often performed using discrete event (DE) simulation. DE simulation of such large systems as MDPS provides challenges in the realization of the simulation itself. Mass data processing of the CERN experiment ALICE Run 3 is, in addition to the large amount of resources required, particularly complex due to the interdependent steps of mass data processing and multi-year duration. Therefore, it is a Long-Term Mass Data Processing (LTMDP). In this paper, an MDPSim simulator for simulating mass data processing will be presented. Finally, a script language for defining a complex workflow in ALICE Run 3 will be described.
\end{abstract}

Keywords: Simulation; MDPSim; CERN; LHC; ALICE

\section{Introduction}

In the last ten years, scientific research has shown an intense increase in the amount and speed of production of data obtained from instruments, detectors, or sensors. One example is the Large Hadron Collider (LHC) [1], whose detectors at CERN produce dozens of PB data per year. [2] The LHC is designed to work with proton beams and heavy ion beams in four large particle physics experiments: ATLAS, CMS, ALICE, and LHCb. The main computational challenge in such scientific research is the computer collection and analysis of data to find evidence of new scientific discoveries.

In the years to come, the LHC is preparing for Run 3 (2021) and after that Run 4 phases of data processing. Predictions show that the amount of data produced and then analysed will increase by 10 (Run 3) to 100 (Run 4) times compared to the current Run 2 phase. For the Run 3 and Run 4 phases of the ALICE experiment, it is planned to collect and process over $100 \mathrm{~PB}$, or $1.000 \mathrm{~PB}$ of data, respectively. In addition to volume, there is a large number of files, so Run 3 provides about 150E6 files in storage systems [3]. The time sequence of execution of the above tasks of the Run 3 computer model is provided by the plan for processing $p p$ and $P b P b$ data, as shown in Figure 1. 


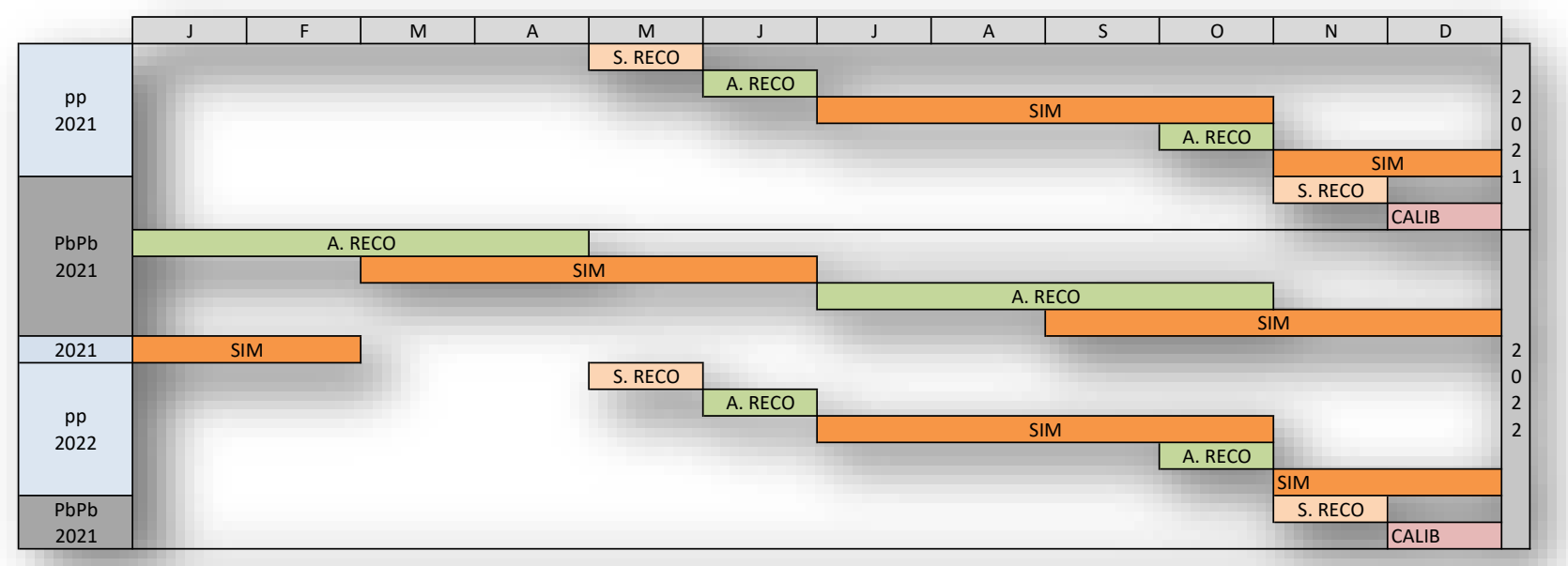

Fig. 1. Data processing plan ALICE Run 3 [4]

Based on the above processing characteristics ALICE Run 3, it is not only about mass data processing (MDP), but also about Long-Term Mass Data Processing (LTMDP). Such dimensioned and long-term data processing requires economic planning and management of large and expensive computer infrastructures. In order to determine the required amount and time sequence of computer resources engagement, one of the standard and precise ways is the simulation of the future system, and simulations of such systems are performed by simulations with discrete events.

A systematic review of simulation tools from HPC, Grid and Cloud systems identified simulation models for mass data processing. In addition to the described characteristics of simulation models for mass data processing, SimGrid and AliEnSim simulators with the most suitable properties of mass data processing stand out. But not fast enough to simulate this type of data processing.[5]

The motivation for this work is that for this level of data processing and the duration and complexity of the workflow, it is necessary and possible to create an efficient simulator for simulating this type of data processing with emphasis on simplicity of workflow description. The purpose of the simulator is to estimate the allocation of computer resources in ALICE Run 3 data processing. Defining a script way of describing tasks for setting long-term data processing that can describe the interaction of planned activities and system capacity; in doing so, the description is parameterized so that changes in the basic parameters of the experiment are directly reflected in the simulated topology and workflow parameters. In this paper, the components of the MDPSim (Mass Data Processing Simulator) simulator for simulating this type of data processing will be presented, as well as the script way for defining the isolated complex $\mathrm{PbPb}$ data processing.

\section{Design and development of Mass Data Processing models}

To determine the quantitative and temporal allocation of resources and to determine the efficient scheduling of processing on allocated resources, in mass data processing systems (MDPS) of the order of ALICE Run 3 it is necessary to design and develop an appropriate simulation model. In a series of simulations, it is possible to improve the efficiency of processing by analysing the results, i.e. to minimize the allocation of resources, and to complete all processing within the planned deadline. To achieve this goal, the first step is to define the components of the simulation model and the associated parameters to simulate a complex workflow such as ALICE Run 3. A top-down approach was used to develop the components of the simulation model.

Only one currently available sufficiently fast developed DE simulation engine Omnet++ was selected for the DE simulation basis [6]. It is based on the $\mathrm{C}++$ core and has become a standard simulator in many simulation domains. Besides, basic omnetpp.ini configuration file within Omnet++ simulation tool is inadequate to describe the complex workflow making use additional files from the applied XML syntax. The file applies a parameterized size definition (eg detector frequency) so that not all sizes change consequently.

Given the requirements of the ALICE Run 3 experiment, a MDPSim model consisting of five basic components was designed: DataCenter, Job Queue, Task generator, File Catalog and NetFlow, as shown in Figure 2. The following part of the paper will describe the components of MDPSim and their application in the ALICE Run $3 \mathrm{PbPb}$ workflow. 


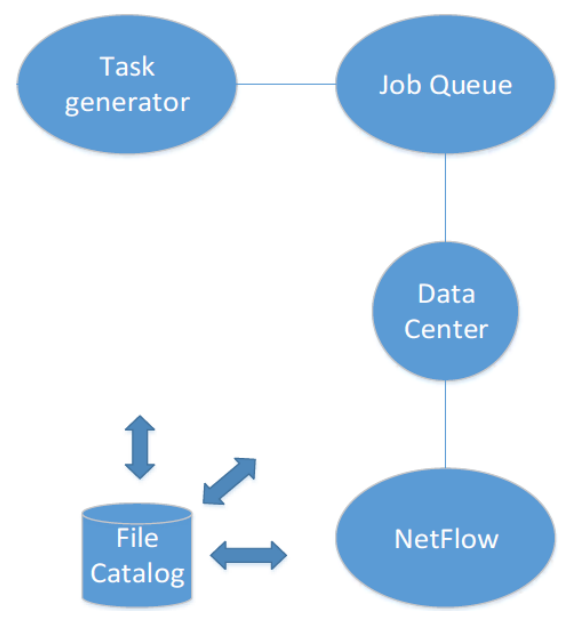

Fig. 2. MDPSim components

\subsection{DataCenter - processing capabilities}

DataCenter component can have two types of computing resources (Computing node and Storage node). Computing node is abstracted by a series of working nodes (slots), usually implemented as a cluster of nodes of the same configuration with the definition of bandwidth both at the level of an individual slot and at the aggregate level of the cluster. Storage node uses storage infrastructure as described in [7], [8], [9], [10]. An example of data center definition (XML language) in a simulation is shown in Figure 3.

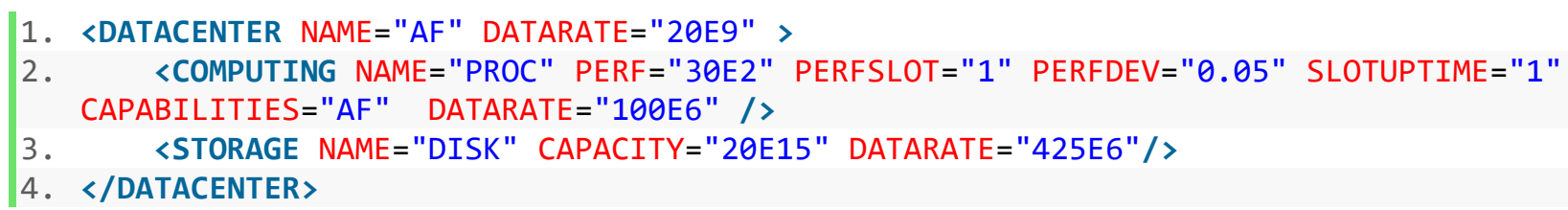

Fig. 3. Example of the DATACENTAR definition in MDPSim format

Data center AF has a symmetric transfer rate (DATARATE) of $20 \mathrm{~GB} / \mathrm{s}$. It consists of two elements:

- computer node (COMPUTING) called PROC with a computer power (PERF) of 300E2 HS06, (PERFSLOT) per slot 1 HS06 (resulting 30.000 slots) with a deviation of execution time of +/- 5\% (PERFDEV), the time difference from the start of the first and last slot from the data center (SLOTUPTIME), the type of computer node capability (CAPABILITIES) and the internal transfer rate of the computer node (DATARATE)

- storage node (STORAGE) called DISK with a capacity (CAPACITY) of $20 \mathrm{~PB}$, with a read/write speed of $425 \mathrm{MB} / \mathrm{s}$ (DATARATE)

\subsection{File Catalog}

The number of files in ALICE computing model is too high ( $>150 \mathrm{E} 6)$ to be simulated using a record for every single file. Actually, the real file catalog requires a clustered database to run smoothly. This simulation is intended to run using only one machine and the file catalog operations should utilize only a fraction of the computer resources. Therefore, the simulation uses a simplified file catalog. It does not operate at the level of every file instance but with the distribution of the file group instances in storage elements. This abstraction reduces the number of possible simulation cases, especially simulations of subtle file replication strategies. The file catalog is implemented using an embedded SQL-compatible database.

\subsection{Job Queue}

Job workload management system is usually implemented using distributed web services that process jobs from inserting into the global job queue to returning results to user. The resulting model is a general job scheduler, i.e. a simulation element called Job Queue, and has the following roles:

- user job analysis and conversion of user specifications into job requirements.

- matching job requirements with resource capabilities

- scheduling sub-jobs in the order defined by job priority classes.

- supervision of work execution. 


\subsection{Task generator}

Task generator simulates a complex process that defines the schedule and type of jobs performed in the system. In addition to the simulation of simple workflow management, the user is also included as the main initiator of the work.

\subsubsection{Input and output data set of task generator}

For the large data volumes as in LHC experiments one of the usual requirements is to process data close to storage. The INDATASET parameter is used to define the input data set that the task will process in the XML file. Parameter syntax:

\section{FILECLASS @ STORAGENAME, COUNT, ACCESS TYPE}

FILECLASS - data type (data type: string)

STORAGENAME - the name of the storage system (data type: string)

COUNT - number of files for processing (data type: integer)

ACCESS TYPE - type of access (value: GLOBAL or LOCAL). If this parameter element is omitted, LOCAL is assumed.

Some examples of use INDATASET:

1. CTF_PbPb@T0::DISK, ALL

Download all (ALL) CTF_PbPb data types stored locally on a T0 disk storage system (DISK).

2. CTF_PbPb@T*::DISK, PbPbCount, LOCAL

Download PbPbCount number of CTF_PbPb data types from all local (LOCAL) T disk storage systems (DISK).

Saving the processed data when setting the task (TASKGEN) in an XML file is represented by the OUT parameter. Parameter syntax:

\section{FileClassName1|share1@SEName1::StorageName1}

FileClassName1 - data type (data type: string).

share1 - defines sharing all COPY/MOVE files on an appropriate storage system (data type: integer). If left out „|share1“ element, it means saving all files to the specified storage system.

SEName1 - the name of the storage system (data type: string).

StorageName1 - type of storage system (data type: string).

Some examples of using the OUT parameter:

1. CTF_PbPb@DS::DISK

Save files type CTF_PbPb data to disk type (DISK) DS storage system.

2. *@SE_T0::TAPE

Saving all (*) data types to SE_T0 tape storage system (TAPE).

\subsection{NetFlow}

NetFlow component simulates the data transmission channel (network internal channels of the device). The MDP is used as a directional weight graph having a set of vertices, connected by one-way or two-way edges. Each data stream inserted into the data transfer simulator is routed using a minimum weight path through active data flow channels. A key condition of the data transmission model is the maintenance of accurate absolute aggregates of performance metrics of data sources and destinations. The file transfer simulation is modelled to be accurate to maintain absolute aggregate measured performance (network bandwidth availability), at the roughest time scale that still provides results usable for system sizing.

\section{Workflow definition}

For the particular experiment like ALICE Run $3 \mathrm{PbPb}$, detector operating frequency is the parameter that defines size and number of output files and following data processing. As detector is under constant improvement process it is not known what the final operating frequency will be. Therefore, it is necessary to simulate different resulting workloads. This required to include parametric definition of simulator components.

The previous chapter 3 describes some the components of the simulator with the corresponding parameter values for defining an individual component in XML format. The following will describe other parameter values in XML format for describing the workflow by individual sections. 


\subsection{EXPR \& STR (expressions and strings)}

Expressions (EXPR) and strings (STR) are used to define complex parameterization schemes for the subsequent processing/resource definition. Expressions and strings are validated from the definition site.

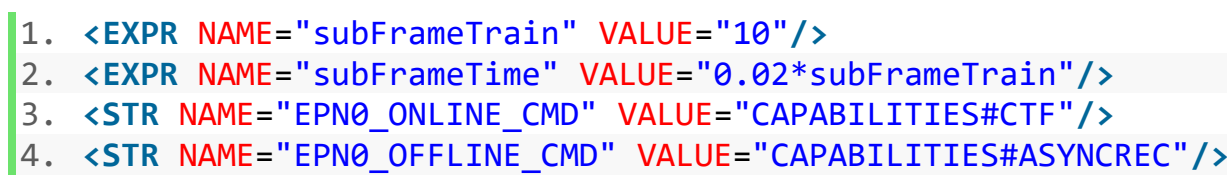

Fig. 4. Example of expressions and strings (EXPR \& STR) description of the workflow in MDPSim format

\subsubsection{EXPR}

The EXPR parameter is used to expression definition. The attributes of the EXPR element are:

- NAME: variable name to hold expression results (data type: string)

- VALUE: can use predefined variables (data type: arithmetic expression).

The definition of a consecutive expression overrides the previous definition. The arithmetic expression evaluator is based on the expressions library [11]. The syntax of the supported expression is similar to C (no bit operations).

The following figures show examples of defining individual quantities in the workflow using EXPR parameters.

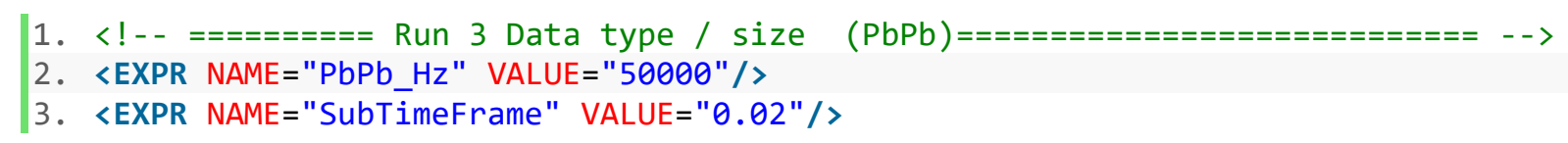

Fig. 5. Detector frequency for $\mathrm{PbPb}$ type of collision using EXPR parameters

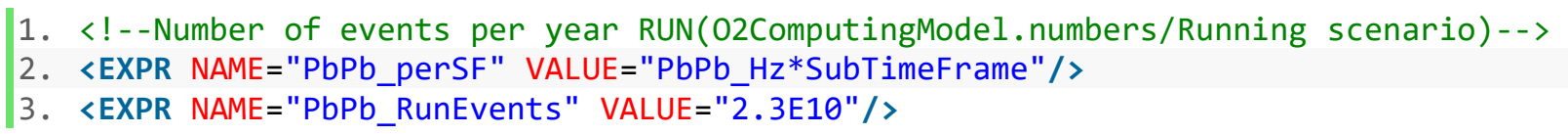

Fig. 6. Number of events for $\mathrm{PbPb}$ type of collision using the EXPR parameter

1. <!-number of jobs per detector RUN(O2ComputingModel.numbers/Storage resources)-> 2. 〈EXPR NAME="PbPb_jobs" VALUE="PbPb_RunEvents/PbPb_perSF"/>

Fig. 7. Defining the number of jobs for PbPb type of collision using EXPR parameters

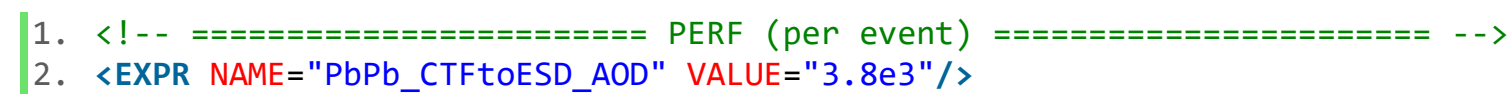

Fig. 8. Defining the required processing power for asynchronous reconstruction in $\mathrm{PbPb}$ collisions using the EXPR parameter

\subsubsection{STR}

The STR parameter is used to define a string. The attributes of the STR parameter are:

- NAME: parametric name (data type: string)

- VALUE: parameter value (data type: string).

A consecutive string definition overrides the previous definition. Figure 9. shows an example of using a STR expression when defining a tag (TAGPREFIX) and the exact date and time (START_TIME) of processing. 


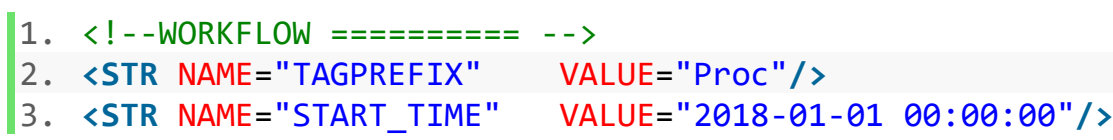

Fig. 9. Defining tag (TAGPREFIX), date and time of the start (START_TIME) of the workflow using STR parameter

\subsection{Definition of processing files}

Within a cluster, instances of files of the same type are affected, which can be on different storage systems. An example of defining a $\mathrm{PbPbCTF}$ class file in a $\mathrm{PbPb}$ collision is shown in Figure 10.

〈FILECLASS NAME="PbPbCTF" SIZE="50000*PbPbPerSF">

Fig. 10. Example of the FILECLASS description in MDPSim format

An example of creating (OPTYPE $=$ "CREATE") files (PbPbJobs), type PbPbCTF, mark 2020 on the storage system T0 :: DISK with the beginning of creation (2020-01-01) is shown in Figure 11.

<FILEINST INSTANCES="PbPbCTF|PbPbJobs, 2020@T0: :DISK" TIME="2020-01-

01" OPTYPE="CREATE"/>

\subsection{Preprocessing}

Fig. 11. Example of the FILEINST description in MDPSim format

Preprocessing can be used with \#define sections to define XML code that can later be included with \#include.

Possible use cases:

- avoiding duplicate XML code (ie T1 layer processing capability)

- use with redefining parameter or repetitive monthly processing tasks can be defined once and then switched on several times (for each month)

Figure 12. shows an example of defining a T1 processing layer of 10.000 slots, general capability, a transfer rate (DATARATE) of $500 \mathrm{~GB} / \mathrm{s}$, a data rate per stream (DATARATESTREAM) of $10 \mathrm{~GB} / \mathrm{s}$ and a processing power (HSO6) per slot of 100, as well as inclusion in a particular part of an xml document.

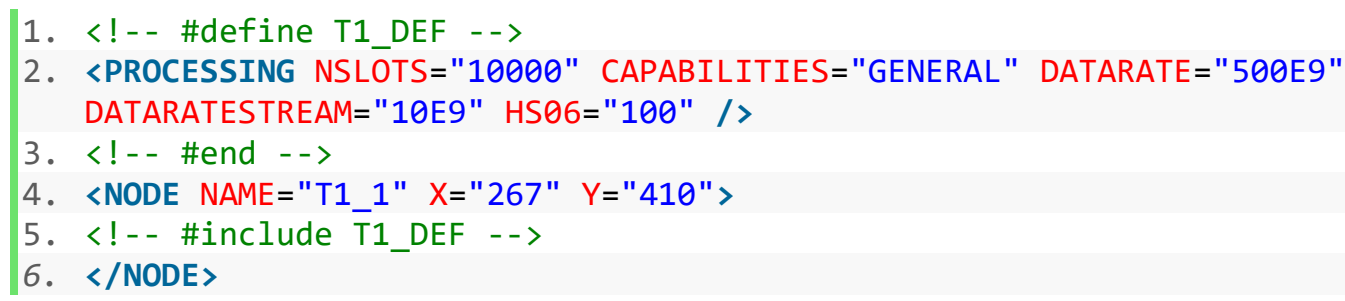

Fig. 12. Defining and including code snippets in an XML file

\subsection{COMMAND}

COMMAND parameter is used to change the capability of processing nodes at a defined time or to enable/disable processing nodes. Parameter syntax:

<COMMAND TIME="yyyy-mm-dd hh:mm" CMD="command@processingNodeMatch1"/>

Examples of applying commands to processing nodes (processingNodeMatch1):

1. IN - inclusion of T0 data center processing nodes on May 1, 2021 at 08:00:00:

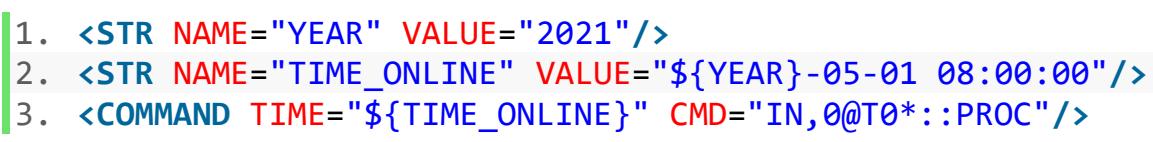

Fig. 13. Defining the year (YEAR), date and time (TIME_ONLINE) to turn on processing nodes using the 
2. OUT - exclusion of T1 data center processing nodes on June 1, 2021 at 08:00:00:

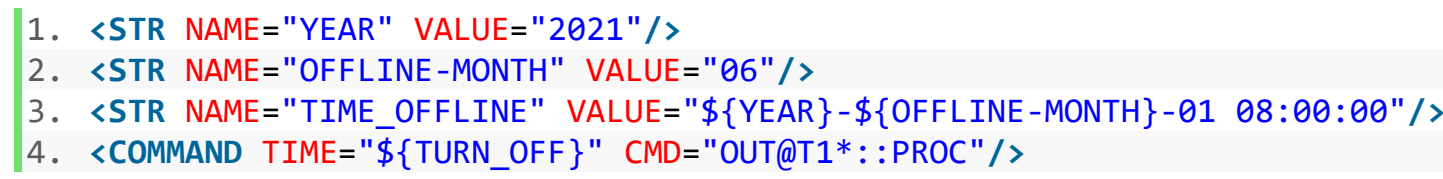

Fig. 14. Defining the year (YEAR), date and time (TIME_OFFLINE) to turn off processing nodes using the

COMMAND parameter

3. CAPABLITIES (redefining the processing capability at a defined time) - setting the processing node capability PN01 to REC value June 1, 2021 at 08:00:00:

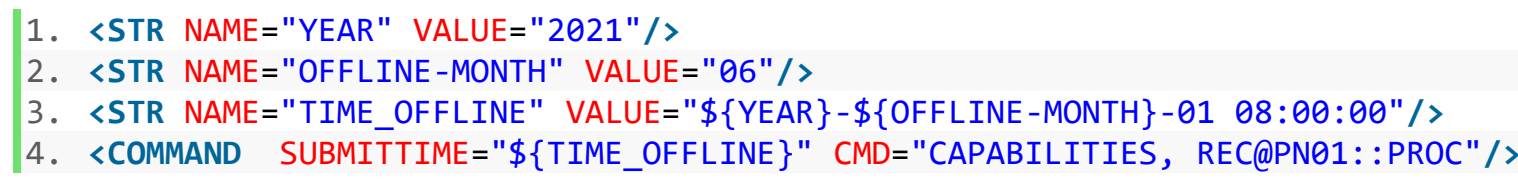

Fig. 15. Defining the year (YEAR), date and time (TIME_OFFLINE) to redefine the processing nodes using the COMMAND parameter

4. PERF (change of processing node performance) - setting for perf value 110.000 and perf per slot (PERFSLOT) 17.5 on processing nodes PN01::PROC

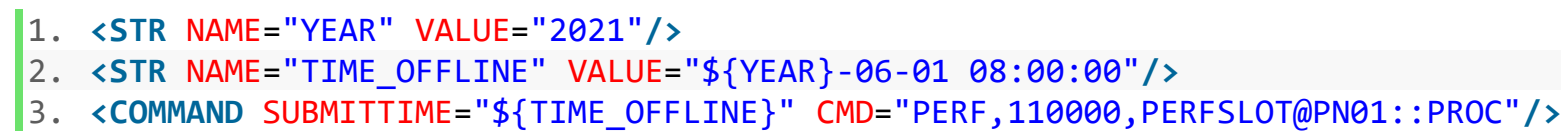

Fig. 16. Defining the year (YEAR), date and time (TIME_OFFLINE) to change the performance of the processing node using the COMMAND parameter

\section{Conclusion}

Mass data processing systems (MDPS) are characterized by the transfer and processing of large amounts of data contained in a large number of files, using complex workflows, all of which require the allocation of large amounts of distributed and heterogeneous computing resources. Among the prominent realized examples of MDPS, CERN's experiments stand out. In the next phase of the collision, ALICE Run 3 raises even more the scale of challenges in terms of workflow complexity, the amount of data and the resulting amount of computing resources required. Discrete event (DE) simulation is used to calculate the required computing resources for these types of problems.

Given the processing characteristics of ALICE Run 3, it is not only about mass data processing, but also about LongTerm Mass Data Processing (LTMDP). The simulation models used so far, which could simulate long and complex workflows, are not fast enough, so a top-down approach was used in the development of simulation models for LTMDP such as ALICE Run 3. For this purpose, the MDPSim simulator was developed using the currently available fastest OMNet++ simulation environment. Besides, for defining a complex workflow, a script way of defining individual system elements has been designed. The MDPSim simulator was used for the simulation an ALICE Run 3 workflow to assess the allocation of computer resources, with a simple script way to describe the workflow. Only parts of the script description method of $\mathrm{PbPb}$ data processing are highlighted in the paper.

In future work, using the developed simulation models, the properties of different processing scenarios will be analyzed in detail. In some even more extreme treatments compared to ALICE Run 3, such as the next ALICE Run 4 phase.

\section{References}

[1] Large Hadron Collider (LHC), Available from: http://lcg.web.cern.ch/LCG/ Accessed: 2018-12-06

[2] Celar, S., Mudnic, E. \& Seremet, Z. (2016). State-of-the-art of messaging for distributed computing systems, Ann. DAAAM proc. 27 
[3] ALICE Collaboration. Upgrade of the ALICE Experiment: Letter Of Intent. CERN-LHCC-2012-012/LHCC-I-022. 2012. Available from: http://cds.cern.ch/record/1475243/ Accessed: 2018-12-16

[4] Buncic, P. (2018). O2 computing and data storage needs, Available from: https://indico.cern.ch/event/775491/contributions/3223484/attachments/1756490/2854965/LHCC-O2-PDP-201812-11-1.pdf Accessed: 2018-12-17

[5] Seremet, Z. (2020). Simulation model for resource allocation for mass data processing, Ph.D Thesis, Department of computing architecture and operating systems, University of Split, Croatia

[6] OMNeT++ Discrete Event Simulator, Available from: https://omnetpp.org/ Accessed: 2018-02-06

[7] XrootD (2018). Available from: http://xrootd.slac.stanford.edu/ Accessed: 2018-03-02

[8] Peters, A. J. \& Janyst, L. (2011). Exabyte Scale Storage at CERN, J. Phys.: Conf. Series 331052015 , doi:10.1088/1742-6596/331/5/052015

[9] CERNBox (2018). Available from: http://cernbox.cern.ch Accessed: 2018-02-26

[10] Seremet, Z.; Celar, S. \& Mudnic, E. (2018). Next Generation Data Flow and Storage Solution in ALICE Experiment, 3rd International Conference on Smart and Sustainable Technologies (SpliTech), Split, 2018, pp. 1-4. Available from: http://ieeexplore.ieee.org/stamp/stamp.jsp?tp=\&arnumber=8448359\&isnumber=8448303 Accessed: 201805-06

[11] Expressions, Available from: https://github.com/Shootfast/expressions, Accessed: 2018-02-01 